Pacific Journal of Mathematics

[WEAKLY] COMPACT OPERATORS AND PF SPACES 


\title{
[WEAKLY] COMPACT OPERATORS AND DF SPACES
}

\author{
WOLFGANG RUESS
}

\begin{abstract}
This is a study of (spaces of) [weakly] compact linear operators with ranges in Fréchet spaces. Characterizations of such operators, extensions and refinements of Schauder's and Gantmaher's Theorems, and results on the approximation property of the space $K(X, Y)$ of compact linear operators are given, together with applications to [weakly] compact operators on function spaces with the strict topology of $R$. C. Buck. Finally, a new tensor product representation for $K^{*}(X, Y), X$ and $Y$ Banach, is established, and compact sets of compact operators on Banach spaces are characterized. The main tools are extensions of Grothendieck's DF techniques.
\end{abstract}

Introduction. This paper is devoted to a study of (spaces of) compact and weakly compact linear operators with ranges in Fréchet spaces. The class of domain spaces is specified to be a class of generalized DF spaces ( $\mathrm{gDF}$ ), which, besides its classical ancestors (and thus all normed spaces), includes the duals of Fréchet spaces under various of the common polar topologies, as well as all function spaces with a strict-like topology as first introduced on spaces of bounded continuous and of bounded holomorphic functions by R. C. Buck [4].

Among the results are an extension and refinement of Schauder's and Gantmaher's Theorems on the [weak] compactness of a linear operator and its adjoint ( $\S 3$, Theorems 3.1 and 3.2), a new tensor product representation for the space $K_{b}(X, Y)$ of compact operators and its dual $K^{*}(X, Y), X$ and $Y$ Banach ( $(33$, Theorem 3.4), or, more generally, $X \mathrm{gDF}$ and $Y$ Fréchet ( $\S 3$, Theorem 3.3), characterizations of operator norm compact sets of compact operators ( $\S 4$ ), and a proof of the approximation property for spaces of compact operators (§1, Theorem 1.14).

The principal tools are extensions of Grothendieck's classical DF space techniques to the wider class of gDF spaces (generalized DF): $A$ locally convex space $X$ is gDF, whenever (1) its strong dual is Fréchet, and (2) its topology is localizable on the bounded sets, i.e. linear operators into other locally convex spaces are continuous as soon as their restrictions to the bounded sets are. Generally speaking, "all" DF properties carry over to gDF spaces. The primary object of $\S 1$ is to verify this for two of the most fruitful DF properties, for which it has been an open problem. Extending the respective 
DF results of Grothendieck [16, I. 1, Thm. 2, p. 64] and [18, I, 1.3, Prop. 5, p. 43], it is shown that (a) hypocontinuity implies continuity for bilinear forms on the product of two gDF spaces, and that (b) gDF spaces solve Grothendieck's "Problème des Topologies" [18, I, 1.1, p. 33]. Theorems 1.4 and 1.9 in $\S 1$ contain the precise (partly more general) statements. These two results are the basic tools for this paper. Also, they answer the corresponding problem of [26, Problème 2] in the affirmative.

Notation and terminology. As far as duality theory for locally convex spaces is concerned, the terminology is that of J. Horváth's book [23] with the following exceptions: For $(X, \tau)$ a locally convex space (abbreviated by "lcs"), $X_{c}^{\prime}, X_{w c}^{\prime}$ and $X_{b}^{\prime}$ denote the topological dual space $X^{\prime}$ of $(X, \tau)$ with the topology of uniform convergence on the compact, the weakly compact and the bounded disks in $(X, \tau)$, respectively. (A disk is a convex circled set.) Accordingly, $X_{c}$ and $X_{w c}$ denote the original space $X$, endowed with the topology of uniform convergence on the compact and the weakly $=\sigma\left(X^{\prime}, X^{\prime \prime}\right)$ compact disks in $X_{b}^{\prime}$, respectively. In particular, the $w c$-topology on $X$ is just the restriction onto $X$ of the Mackey topology $\tau\left(X^{\prime \prime}, X^{\prime}\right)$ of $X^{\prime \prime}$ with respect to $X^{\prime}$. As usual [23] the Mackey topology of $(X, \tau)$ itself (uniform convergence on the weak ${ }^{*}=\sigma\left(X^{\prime}, X\right)$-compact disks in $\left.X^{\prime}\right)$ is denoted by $\tau\left(X, X^{\prime}\right)$.

The convex circled hull of a subset $A$ of a linear space $X$ will be denoted by $\Gamma A$.

The space of continuous linear operators from an les $X$ into an lcs $Y$ is denoted by $L(X, Y)$, the space of continuous bilinear forms from $X \times Y$ into $K$ by $B(X, Y)$.

An operator $u \in L(X, Y), X$ and $Y$ lcs, is called [weakly] compact, if there exists a zero neighbourhood $U$ in $X$ such that $u(U)$ is [weakly] relatively compact in $Y$.

The space of compact linear operators from an les $X$ into an les $Y$ is denoted by $K(X, Y) . L(X, Y)$ and $K(X, Y)$ will always be assumed to be endowed with the topology of uniform convergence on the bounded subsets of $X$ (=operator norm in case $X$ and $Y$ are normed), as being indicated by $L_{b}(X, Y)$ and $K_{b}(X, Y)$.

1. Extensions of Grothendieck's DF techniques. This section is devoted to a discussion of the extension of the DF techniques to the following wider class of locally convex spaces:

Definition 1.1. [24, 25], [32, 34]: An les $(X, \tau)$ is called gDF (generalized DF space), if (1) its strong dual $X_{b}^{\prime}$ is a Fréchet space, and (2) its topology is localizable on the bounded sets, i.e. linear 
operators into other locally convex spaces are continuous as soon as their restrictions to the bounded sets are.

Equivalently, $(X, \tau)$ is $\mathrm{gDF}$, whenever it has a fundamental sequence $\left(B_{n}\right)_{n \in N}$ of bounded sets (every $B$ bounded in $(X, \tau)$ is absorbed by some $B_{n}$ ), and $\tau$ is the finest locally convex topology on $X$ that agrees with $\tau$ on the $B_{n}$ 's, $n \in N$.

\section{EXAMPLES.}

1. All DF spaces [16, Déf. 1, p. 63] are gDF. In particular, strong duals of metrizable les.

More generally:

2. Let $X$ be a metrizable les. Then its strong dual (uniform convergence on the bounded subsets of $X$ ), its $c$-dual $X_{c}^{\prime}$ (uniform convergence on the precompact subsets of $X$ ), and in case $X$ is Fréchet, also its Mackey dual $X_{w c}^{\prime}$ (uniform convergence on the weakly compact disks in $X$ ) are gDF $[32,34]$.

3. Accordingly, whenever $X$ is an les whose strong dual $X_{b}^{\prime}$ is Fréchet, then $X_{c}$ (uniform convergence on the compact subsets of $X_{b}^{\prime}$ ) and $X_{w c}$ (uniform convergence on the weakly $=\sigma\left(X^{\prime}, X^{\prime \prime}\right)$-compact subsets of $X_{b}^{\prime}$ ) are $\mathrm{gDF}$, see Proposition 2.6 in $\S 2$.

4. R. C. Buck's strict topology $\beta$ on $C_{b}(S)$ [4], $S$ locally compact Hausdorff, and its various extensions to (i) $C_{b}(T), T$ completely regular [11, 40], (ii) Banach modules over Banach algebras [42], and (iii) the double centralizer algebra of a $C^{*}$-algebra [5], all these "strict" spaces, in general, are far from being DF but, again, turn out to be gDF. (Consequences of this observation for such function spaces have been the point of discussion of the paper [33]; see also the survey [34].)

Further examples in this context are F. D. Sentilles' [41] strict topology $\beta$ on $L^{\infty}$ in his $L^{\infty}$ - $L^{1}$-duality, and the "universal strongly countably additive" topology $\tau$ on the space $\mathscr{S}(\mathscr{R})$ (of simple functions on a ring $\mathscr{R}$ of subsets of a set $S$ ) of W. H. Graves [13] in his representation of strongly countably additive vector measures (on $\mathscr{R}$ ) as continuous linear operators (on $(\mathscr{S}(\mathscr{R}), \tau)$ ).

Applications in the context of strict topologies will eventually be pointed out in this paper.

These examples show that gDF spaces considerably enlarge the class of DF spaces, and include many more spaces of analysis. The interesting fact to note now, and the important one for our discussion, is that, nevertheless, they still have all the nice DF properties.

Notes 1.2. (1) The gDF spaces as defined here have first been 
introduced by $K$. Noureddine $[24,25]$ as "espaces $D_{b}$ "; the semiMontel ones among them appear under the name "dF" in $\mathrm{K}$. Brauner [3], and under the name "DCF" in Hollstein [21], who also considered non-locally convex analogues [20].

(2) Noureddine $[24,25]$ already showed that gDF spaces share many properties with the DF spaces. For later use, the following are noted here:

(a) [24, Cor. 1 and 2]: If $A$ is a gDF subspace of an lcs $X$, then every bounded subset of the closure of $A$ in $X$ is contained in the closure of a bounded subset of $A$. In particular, a gDF space is complete if and only if it is quasi-complete (i.e. closed bounded sets are complete).

An extension of these results to les with a fundamental sequence of bounded sets and the property that strong nullsequences in their dual are equicontinuous, has been given in [33, Cor. 2.4].

(b) [25, Thm. 3.1.7]: (cnc) "countable neighbourhood condition": For every sequence $\left(U_{n}\right)_{n \in N}$ of zero neighbourhoods in a gDF space, there exists a sequence $\left(\alpha_{n}\right)_{n \in N}$ of positive real numbers such that $U=\cap\left\{\alpha_{n} U_{n} \mid n \in N\right\}$ again is a zero neighbourhood.

(c) [25, Thm. 1.1.7]: Relatively compact subsets of the strong dual of a $\mathrm{gDF}$ space are equicontinuous.

In particular, gDF spaces are sequentially evaluable:

Definition [44]: An les $X$ is called sequentially evaluable if every strong nullsequence in its dual is equicontinuous.

(3) Further DF properties have been carried over to gDF spaces in [10] and [33].

(d) [10,33]: gDF spaces are quasinormable (see Definition 1.3 below).

It seems worth noticing at this point that, for the special case of the gDF space $X_{w c}^{\prime}$ for $X$ Fréchet (Examples 2), property (d) directly translates into the following result:

LemMa. For every weakly compact disk $B$ in a Fréchet space $(Y, \rho)$, there exists another such, $C$ say, with the property that $B \subset C$ and that the norm $q_{C}$ generated by $C$ on $Y_{C}=\operatorname{span}(C)$ induces on $B$ the same topology as $\rho$. In particular, $B$ is a weakly compact disk in (the Banach space) $\left(Y_{C}, q_{C}\right)$. 
The compact analogue of this result is a well known and widely used consequence of the Banach-Dieudonné Theorem. To my knowledge, the above weakly compact version is not to be found explicitly anywhere in the literature, whereas the existence of a bounded $C$ with the indicated properties [18, I, 4.1, Lemme 10, p. 105] is very well being used.

(e) [33]. A gDF space, or, more generally, a sequentially evaluable les with a fundamental sequence of bounded sets, is nuclear, if and only if its strong dual is nuclear.

(4) Two of the most important and fruitful DF properties remained open for $\mathrm{gDF}$ spaces. For DF spaces, Grothendieck had shown:

(f) [16, I, Thm. 2, p. 64]: Equihypocontinuous sets of bilinear mappings on the (cartesian) product of two DF spaces are equicontinuous.

(g) [18, I, 1.3, Prop. 5, p. 43]. "Problème des Topologies": For two DF spaces $X$ and $Y$, their projective tensor product $X \otimes_{\pi} Y$ and its completion again are $\mathrm{DF}$. On the space $B(X, Y)$ of continuous bilinear forms on $X \times Y$, the topology of bibounded convergence is equal to the strong topology of the dual of $X \tilde{\otimes}_{\pi} Y$, i.e., every bounded subset of $X \widetilde{\otimes}_{\pi} Y$ is contained in the closed absolutely convex hull of a set $A \otimes B, A$ bounded in $X, B$ bounded in $Y$.

Noureddine [26, Thms. 2 and 3] proved (g) for semi-Montel gDF spaces and left the general case as a problem [26, Problème 2, p. 103]. Satz 2.1 of [20] yields (f) for gDF spaces. The nonlocally convex results of [20] include the second statement of (g) for gDF spaces, whereas it is not evident to me, whether this also extends to the first one.

Proposition ( $f$ ) will now be proved for a much wider class than the gDF spaces [32, II. 4, Satz 4.11, and IV. 2, Satz 2.1], and proposition (g) for $\mathrm{gDF}$ spaces [32, II. 4, Satz 4.8 and Satz 4.9] will then follow easily.

As a final result, it is now settled, that all important $\mathrm{DF}$ properties, except the one of being countably evaluable, remain valid for $\mathrm{gDF}$ spaces. It is for this reason that $I$ chose (in $[32,34]$ ) to change the original terminology of Noureddine and to let their close relationship with their ancestors show through this different name.

Recall the following notions:

DEFINITION 1.3.

1. An les $X$ is called quasinormable [16, III. 1, Def. 4, p. 106], 
whenever, for every equicontinuous subset $H$ of the dual of $X$, there exists a zero neighbourhood $U$ in $X$ such that, on $H$, the strong topology and the topology of uniform convergence on $U$ coincide.

Equivalently, $X$ is quasinormable, whenever, for every zero neighbourhood $U$, there exists another such, $V$ say, with the property that, for every $\varepsilon>0$, there exists a bounded subset $B_{\varepsilon}$ of $X$ such that $V \subset \varepsilon U+B_{\varepsilon}$.

Also recall, that a Schwartz space exactly is a quasinormable lcs whose bounded sets are precompact.

2. Given an les $(X, \tau)$ with an increasing sequence $A=\left(A_{n}\right)_{n \in N}$ of disks, $\tau$ is said to be localizable on the $A_{n}$ 's, whenever $\tau$ is the finest lc topology on $X$ which agrees with $\tau$ on the $A_{n}$ 's.

In case the union of the $A_{n}$ 's spans $X$, and $A_{n}+A_{n} \subset A_{n+1}$, a base of zero neighbourhoods for the finest lc topology on $X$, agreeing with $\tau$ on the $A_{n}$ 's is formed by the absolutely convex hulls of sets of the form $\cup\left\{U_{n} \cap A_{n} \mid n \in N\right\},\left(U_{n}\right)_{n \in N}$ a sequence of $\tau$-zero neighbourhoods [12, Prop. 1].

3. Given three les $X, Y$ and $Z$, a set $H$ of bilinear maps from $X \times Y$ into $Z$ is called equihypocontinuous, whenever, for every $B$ bounded in $X$, the set $H(B, \cdot)$ is an equicontinuous subset of $L(Y, Z)$, and the set $H(\cdot, C)$ is an equicontinuous subset of $L(X, Z)$ for every $C$ bounded in $Y$.

\section{THEOREM 1.4 .}

(1) A set of bilinear maps from the product $X \times Y$ of two $\mathrm{gDF}$ spaces $X$ and $Y$ into an les $Z$ is equicontinuous, if and only if it is equihypocontinuous. More generally, the following statement holds:

(2) Let $X$ be a quasinormable lcs and $(Y, \rho)$ an lcs. Whenever either

(i) $Y$ contains an absorbing disk $B(\operatorname{span}(B)=Y)$ such that $\rho$ is localizable on $B$, or

(ii) $Y$ contains an increasing sequence $\left(B_{n}\right)_{n \in N}$ of disks such that $\rho$ is localizable on the $B_{n}$ 's, and $X$ fulfills condition (cnc) of proposition (b) of Notes 1.2 above, or

(iii) $(Y, \rho)$ has a fundamental sequence $\left(B_{n}\right)_{n \in N}$ of bounded sets and fulfills (cnc), and $X$ is $\mathrm{DF}$, then a set of bilinear maps from $X \times Y$ into an les $Z$, which is equihypocontinuous with respect to the bounded subsets of $X$ and the set $B$ (resp. the $B_{n}$ 's) in $Y$, is equicontinuous.

Notes. (1) Proposition (iii) is to be found in $[16$, I. 1, Thm. 2, p. 64, and Rem. 2, p. 66].

(2) Proposition (i) seems to be the first result in the nonmetrizable context that dispenses completely with the assumption of 
a fundamental sequence of bounded sets for one of the factors. It includes the following particular cases:

CoROllary 1.5. Let $X$ be a quasinormable lcs. Then every equihypocontinuous set of bilinear maps from $X \times Y$ into an les $Z$ is equicontinuous, whenever.

(a) $Y$ is any of the "strict" spaces listed among the examples at the beginning of this section, or

(b) for a Banach space $Z, Y$ is any of the spaces $Z_{c}, Z_{w c}, Z_{c}^{\prime}, Z_{w_{c}}^{\prime}$.

A particular direct consequence of the above results is the following surprising improvement of Theorem 4.12 of [41] (see this paper for terminology and details).

THEOREM 1.6. Multiplication is $\beta$-jointly continuous on $L^{\infty}(\mathscr{A})$ whenever $\beta$ is Hausdorff.

This result, in turn, yields immediate proofs of Prop. 8.7 and Thm. 8.8 of [41] on the "Radon-Nikodym-map" $P_{\hat{v}}$, for

$$
\hat{v} \in L^{1}: P_{\hat{v}}: L^{\infty}(\mathscr{A}) \longrightarrow L^{1}(\mathscr{A}), f \longmapsto f \cdot \hat{v},
$$

where, for $g \in L^{\infty}(\mathscr{L}), f \cdot \hat{v}(g):=\hat{v}(f \cdot g)$. Since $\hat{v} \in L^{1}(\mathscr{A})$ is $\beta$-continuous, there exists a $\beta$-zero neighbourhood $V$ in $L^{\infty}(\mathscr{L})$ on which $\hat{v}$ is bounded by one in absolute value. $\beta$-continuity of multiplication now asserts the existence of a $\beta$-zero neighbourhood $U$ in $L^{\infty}(\mathscr{A})$ such that $U \cdot U \subset V$. In terms of $P_{\hat{v}}$ this yields $P_{\hat{v}}(U) \subset U^{0}$. In particular, $P_{\hat{v}}$ is $\beta-\|\|_{1}$-continuous. In case the dual of $\left(L^{1}(\mathscr{A}),\|\|_{1}\right)$ is equal to $L^{\infty}(\mathscr{A})$ (consult [41]), it even is weakly compact from $\left(L^{\infty}(\mathscr{A}), \beta\right)$ into $\left(L^{1}(\mathscr{A}),\|\|_{1}\right)$.

(3) $A$ particularly striking application of Theorem 1.4 to sets of [weakly] compact operators is to be found in $\S 2$, see Proposition 2.1 and its proof.

(4) Note that, besides all gDF spaces, the class of spaces that fulfill the assumptions of propositions (2) (i) and (ii) of Theorem 1.4 on $X$, contains all subspaces of Schwartz gDF spaces. This is worth mentioning, for, in general, the gDF property is not inherited by linear subspaces. Note as well that the class of $Y$ 's as specified in (2) (iii) is closed under the formation of linear subspaces.

Proof of Theorem 1.4. We shall prove the following more technical result which, much like Theorem 1.4 itself, provides remarkable consequences for sets of [weakly] compact operators from gDF spaces into Fréchet spaces; see Theorem 2.2 and the Note following the 
proof of this theorem in $\S 2$.

Proposition 1.7. Let $(X, \tau)$ be a quasinormable les with property (cnc), $(Y, \rho)$ an lcs with an increasing sequence $\mathscr{B}=\left(B_{n}\right)_{n \in N}$ of disks whose union spans $Y$. Then a set $H$ of bilinear maps from $X \times Y$ into an les $Z$ is equicontinuous from $(X, \tau) \times(Y, \eta)$ into $Z$, whenever it is $\mathscr{B}_{\tau} \times \mathscr{B}$-equihypocontinuous. (Here, $\eta$ denotes the finest lc topology on $Y$ agreeing with $\rho$ on the $B_{n}$ 's, and $\mathscr{B}_{\tau}$ denotes the class of all bounded subsets of $(X, \tau)$.)

Proof. It suffices to give a proof for the case $Z=K$, see [16, I. 1, Lemme 3, p. 64]. Also, considering $2^{n} B_{n}$ instead of $B_{n}, n \in N$, one can assume that $B_{n}+B_{n} \subset B_{n+1}, n \in N$, for $\eta$ is not being changed by this manipulation.

1. For every $n \in N$, there exists a zero neighbourhood $U_{n}^{\prime}$ in $(X, \tau)$ such that $\left|H\left(U_{n}^{\prime}, B_{n}\right)\right| \leqq 1$ (equihypocontinuity of $H$ ).

2. For every $n \in N$, there exists a zero neighbourhood $U_{n}$ in $(X, \tau)$ with the property that, for every $\alpha>0$, there exists a bounded subset $M_{\alpha}^{n}$ of $(X, \tau)$ such that $U_{n} \subset \alpha U_{n}^{\prime}+M_{\alpha}^{n}$ (quasinormability of $X)$.

3. There exists a sequence $\left(\alpha_{n}\right)_{n \in N}$ of positive reals such that $U=\cap\left\{\alpha_{n} U_{n} \mid n \in N\right\}$ is a zero neighbourhood in $X$ ((cnc) for $\left.X\right)$.

4. For every $n \in N$, there exists a zero neighbourhood $V_{n}$ in $(Y, \rho)$ such that $\left|H\left(M_{\alpha_{n}^{n}}^{n}, V_{n}\right)\right| \leqq 1$ (equihypocontinuity of $H$ again).

It follows that $H\left(U, \bigcup_{m \in N}\left(B_{m} \cap \alpha_{m}^{-1} V_{m}\right)\right) \subset H\left(U_{n}^{\prime}+\alpha_{n} M_{\alpha_{n}^{-1}}^{n}\right.$, $\left.\bigcup_{m \in N}\left(B_{m} \cap \alpha_{m}^{-1} V_{m}\right)\right)$ for all $n \in N$. Hence, by 1. and 4., we have:

5. $\left|H\left(U, \cup\left\{B_{m} \cap \alpha_{m}^{-1} V_{m} \mid m \in N\right\}\right)\right| \leqq 2$.

The set $V=\Gamma \cup\left\{B_{m} \cap \alpha_{m}^{-1} V_{m} \mid m \in N\right\}$ is an $\eta$-zero neighbourhood in $Y$ (see Definition 1.3 above), and, by 5 ., we conclude that $|H(U, V)| \leqq 2$, which completes the proof.

Projective tensor products of gDF spaces are next.

Proposition 1.8. Let $X$ and $Y$ be $\mathrm{gDF}$ spaces with respective fundamental sequences $\left(A_{n}\right)_{n \in N}$ and $\left(B_{n}\right)_{n \in N}$ of bounded sets, all disks, and $A_{n}+A_{n} \subset A_{n+1}$ and $B_{n}+B_{n} \subset B_{n+1}$. Then the projective tensor product topology $\pi$ on $X \otimes Y$ is localizable on the sets $C_{n}=$ $\Gamma\left(A_{n} \otimes B_{n}\right), n \in N$.

A variety of consequences follows:

TheOREM 1.9. ("Problème des Topologies" for gDF spaces); Let $X$ and $Y$ be $\mathrm{gDF}$ spaces with respective fundamental sequences 
$\left(A_{n}\right)_{n \in \mathbf{N}}$ and $\left(B_{n}\right)_{n \in N}$ of bounded sets. Then $X \otimes_{\pi} Y$ and $X \tilde{\bigotimes}_{\pi} Y$ are gDF spaces as well, with fundamental sequences $\left(\overline{\Gamma A_{n} \otimes B_{n}}\right)_{n \in N}$ (closure in the respective space) of bounded sets. In particular, the topology of bibounded convergence (uniform convergence on $A \times B, A$ bounded in $X, B$ bounded in $Y$ ) on the space $B(X, Y)$ of continuous bilinear forms on $X \times Y$ is equal to the strong topology on $B(X, Y)$ as the dual of $X \otimes_{\pi} Y$ and of $X \widetilde{\boldsymbol{\otimes}}_{\pi} Y$.

Proof. Combine Proposition 1.8 and proposition (a) on gDF spaces in Notes 1.2 above with the fact [30, Thm. 4] that, whenever an lc topology is localizable on an increasing sequence $C_{n}$ of bounded disks whose union spans the whole space, its bounded sets are exactly those absorbed by the closures of the $C_{n}$ 's.

Corollary 1.10. For gDF spaces $X$ and $Y$ with respective fundamental sequences $\left(A_{n}\right)_{n \in N}$ and $\left(B_{n}\right)_{n \in N}$ of bounded sets, a set $H$ of linear mappings $X \boldsymbol{\bigotimes}_{\pi} Y$ (resp. from $X \tilde{\boldsymbol{\otimes}}_{\pi} Y$ ) into an les $Z$ is equicontinuous, if and only if $H \mid \Gamma A_{n} \otimes B_{n}$ (resp. $H \mid \overline{\Gamma A_{n} \otimes B_{n}}$ ) is equicontinuous (at 0 ) for all $n \in N$.

Corollary 1.11. For $X$ and $Y$ gDF spaces, every precompact subset of $B_{b b}(X, Y)$ is equicontinuous.

(For semi-Montel gDF spaces, this is Lemme 2 in $\S 4$ of [26]).

Proof of Corollary 1.11. Combine Theorem 1.9 with property (c) of $\mathrm{gDF}$ spaces in Notes 1.2 above.

Corollary 1.12. Let $X$ and $Y$ be gDF spaces.

(i) Whenever both $X$ and $Y$ are Schwartz lcs, then $X \otimes_{\pi} Y$ is Schwartz gDF, and $X \widetilde{\boldsymbol{\otimes}}_{\pi} Y$ is semi-Montel Schwartz gDF.

(ii) Whenever $X$ is Schwartz and $Y$ is semi-reflexive, then $X \widetilde{\bigotimes}_{\pi} Y$ is semi-reflexive gDF.

(For semi-Montel DF spaces, compare [18, I, 1.3, Cor. 2, p. 45]; at this time it was not yet known that $\mathrm{DF}$ spaces are quasinormable.)

THEOREM 1.13. Whenever $X$ and $Y$ are Schwartz gDF spaces with the approximation property (a.p.), then the space $X \tilde{\otimes}_{\pi} Y$ has the approximation property as well.

REMARK. For barrelled Montel DF spaces, this follows from [2, 4, Satz 1, p. 212]. Recall that these spaces are exactly the strong 
duals of Fréchet Montel spaces, whereas the class of Schwartz gDF spaces contains the $c$-duals of any metrizable lcs. This extension is essential for the proof of Theorem 1.14 below.

Proof of Theorem 1.13. The proof is a combination of Theorem 1.9 and a result of [19]. Heinrich's elegant direct proof of the a.p. for $X \tilde{\otimes}_{\pi} Y$ for $X$ and $Y$ Fréchet spaces with a.p. [19, Thm. 3] actually shows that the following result is true. Let $X$ and $Y$ be lcs with the property that every precompact subset $P$ of $X \widetilde{\otimes}_{\pi} Y$ is contained in the closed absolutely convex hull of a set $P_{1} \otimes P_{2}$ for precompact subsets $P_{1}$ of $X$ and $P_{2}$ of $Y$. Then, if both $X$ and $Y$ have a.p., $X \widetilde{\otimes}_{\pi} Y$ has a.p. In case of Theorem 1.13, Theorem 1.9 reveals that the assumptions of this result are fulfilled.

Note. In conjunction with Theorem 3.4 in $\S 3$, Theorem 1.13 yields the well known fact that $K(X, Y)$ has a.p. whenever $X$ and $Y$ are Banach spaces with $X^{*}$ and $Y$ having a.p.: It is folklore (polarity techniques) that a Fréchet space has a.p. if and only if its $c$-dual has a.p. Hence, given $X, Y$ Banach with $X^{*}$ and $Y$ a.p., $X_{c}^{* *}$ and $Y_{c}^{*}$ have a.p. and thus, by Theorem 1.13, the space $X_{c}^{* *} \tilde{\Theta}_{\pi} Y_{c}^{*}$ as well. But, according to Theorem 3.4, this is the $c$-dual of the Banach space $K(X, Y)$. Hence, $K(X, Y)$ has a.p.

But, using Theorem 3.3 instead, much more can be said. The following extension of the classical Banach space result to the gDF$F$-situation holds:

THEOREM 1.14. Let $X$ be a gDF space and $Y$ a Fréchet space such that $Y$ and the strong dual $X_{b}^{\prime}$ of $X$ have the approximation property. Then the Fréchet space $K_{b}(X, Y)$ of compact linear operators from $X$ into $Y$, endowed with the topology of uniform convergence on the bounded subsets of $X$, has the approximation property.

It remains to prove Proposition 1.8: We have to show that $\pi$ is equal to the finest lc topology on $X \otimes Y$, agreeing with $\pi$ on the sets $C_{n}=\Gamma A_{n} \otimes B_{n}$. Denoting this latter topology by $\eta$, and referring to the general properties of the projective tensor product topology, it is enough to show that the tensor mapping $\Phi: X \times Y \rightarrow X \otimes Y,(x, y) \mapsto$ $x \otimes y$, is continuous from $X \times Y$ into $(X \otimes Y, \eta)$. Theorem 1.4 reduces this to hypocontinuity, i.e. that $\Phi\left(A_{n}, \cdot\right)$ and $\Phi\left(\cdot, B_{n}\right)$ are equicontinuous sets of linear operators from $Y$ resp. $X$ into $(X \otimes Y, \eta)$ for all $n \in N$. By symmetry, and according to the fact that $X$ and $Y$ are gDF, it suffices to prove that the restrictions $\Phi\left(A_{n}, \cdot\right) \mid B_{m}$ are equicontinuous at 0 for all $m, n \in N$. This is what we show now. 
Let $m, n \in N$ and $W$ an $\eta$-zero neighbourhood.

(1) There exist sequences $\left(U_{k}\right)_{k \in N}$ and $\left(V_{k}\right)_{k \in N}$ of zero neighbourhoods in $X$ and $Y$, respectively, such that $W \supset \Gamma \cup\left\{\left(\Gamma A_{k} \otimes B_{k}\right) \cap\right.$ $\left.\left(\Gamma U_{k} \otimes V_{k}\right) \mid k \in N\right\}$ (see proposition 2 of Def. 1.3).

(2) For $k \in N$, choose $\alpha_{k}^{n}>0$ such that $A_{n} \subset \alpha_{k}^{n} U_{k}$. Let $j=$ $\max \{n, m\}$, and let $(x, y) \in\left(A_{n} \times\left(B_{m} \cap\left(\alpha_{j}^{n}\right)^{-1} V_{j}\right)\right.$. Then we have:

(3) $x \otimes y \in\left(A_{j} \otimes B_{j}\right) \cap\left(\left(\alpha_{j}^{n} U_{j}\right) \otimes\left(\left(\alpha_{j}^{n}\right)^{-1} V_{j}\right)\right)=\left(A_{j} \otimes B_{j}\right) \cap\left(U_{j} \otimes V_{j}\right)$.

In conjunction with (1), this yields $\Phi\left(A_{n}, B_{m} \cap\left(\alpha_{j}^{n}\right)^{-1} V_{j}\right) \subset W$, which completes the proof.

2. [Weak] Compactness of linear operators. The starting point for our discussions are Grothendieck's classical results [16, Cor. 1 of Thm. 11, p. 114], and [17, IV, 4.3, Cor. 1 of Thm. 2, p. 241]:

Every continuous linear operator from a quasinormable les $X$ into a Banach space $Y$, which transforms bounded sets into [weakly] relatively compact sets, is [weakly] compact.

The following quite recent result of van Dulst is a variation/ extension of the theme [10, Thm. p. 111]:

The conclusion of Grothendieck's result holds, whenever $Y$ is a Fréchet space, and $X$ a quasinormable lcs with (cnc) (see proposition 2 of Definition 1.3 in $\S 1$ ).

Note that every gDF space fulfills the assumptions on $X$.

As a first step towards our characterization of (weakly) compact operators along this line, an extension to sets of (weakly) compact operators of these two results is shown to be an immediate consequence of Theorem 1.4:

Proposition 2.1. Let $H$ be an equicontinuous set of linear operators from an les $X$ into an les $Y$ such that $H(B)$ is [weakly] relatively compact in $Y$ for all $B$ bounded in $X$. If either

(a) $Y$ is Fréchet and $X \mathrm{gDF}$ (or, more generally, a quasinormable les with (cne)), or

(b) $Y$ is Banach and $X$ quasinormable, then there exists a zero neighbourhood $U$ in $X$ such that $H(U)$ is [weakly] relatively compact in $Y$.

Proof. Considering the set $\hat{H}$ of bilinear forms on $X \times Y^{\prime}$ associated to the $h \in H\left(\hat{h}\left(x, y^{\prime}\right):=\left\langle h x, y^{\prime}\right\rangle\right)$, the assumptions on $H$ mean that $\hat{H}$ is equihypocontinuous on $X \times Y_{c}^{\prime}$ (resp. on $X \times Y_{w c}^{\prime}$ ). Hence, in both cases, Theorem 1.4 reveals that $\hat{H}$ is equicontinuous for these topologies. This proves the assertion. 
A further variation of the theme, this time with an additional aspect concerning the topologies of the range space, is F. D. Sentilles' characterization of [weakly] compact operators on the space $\left(C_{b}(S), \beta\right)$ of bounded continuous functions on a locally compact Hausdorff space $S$, endowed with the strict topology $\beta$ of R. C. Buck [4]:

[39, Thms. 2 and 4]. Given two locally compact Hausdorff spaces $S$ and $T$, a linear operator from $\left(C_{b}(S), \beta\right)$ into $\left(C_{b}(T), \beta\right)$ is [weakly] compact, if and only if it is continuous as an operator from $\left(C_{b}(S), \beta\right)$ into $\left(C_{b}(T)\right.$, sup-norm), and transforms $\beta$-bounded (= sup-norm bounded!) subsets of $C_{b}(S)$ into $\beta$-[weakly] relatively compact subsets of $C_{b}(T)$.

As this characterization is not being covered by the above abstract results, it motivated the search for an appropriate extension. Such is provided by the following result, which contains all the results considered so far as special cases.

THEOREM 2.2. Let $X$ be a quasinormable les and $(Y, \rho)$ an lcs with a further locally convex topology $\rho_{1}$, finer than $\rho$. Whenever $H$ is an equicontinuous set of linear operators from $X$ into $\left(Y, \rho_{1}\right)$ and, in addition, either.

(a) $\rho_{1}$ is normable, or

(b) $\rho_{1}$ is metrizable and $X$ has (cnc) (see Notes 1.2, 2.(b)), then the following statements hold:

1. If $H(B)$ is o-precompact in $Y$ for all $B$ bounded in $X$, then there exists a zero neighbourhood $U$ in $X$ such that $H(U)$ is precompact in $(Y, \rho)$.

2. If $H(B)$ is $\rho$-weakly relatively compact in $Y$ for all $B$ bounded in $X$, and if $(Y, \rho)$ is sequentially complete, then there exists a zero neighbourhood $U$ in $X$ such that $H(U)$ is $\rho$-weakly relatively compact in $Y$.

3. If $X$ is a gDF space with fundamental sequence $\left(B_{n}\right)_{n \in N}$ of bounded sets, and if $\left(V_{n}\right)_{n \in N}$ is a (decreasing) zero neighbourhood base for $\rho_{1}$ on $Y$ (all $V_{n}$ disks), then the zero neighbourhood $U$ in $X$ in propositions 1. and 2. above can be chosen to be $U=\cap\left\{n B_{n}+\stackrel{(-1)}{H}\left(V_{n}\right) \mid n \in N\right\}$.

Notes. (a) The additional information on the special zero neighbourhood $U$ in $X$ as given in part 3 is particularly useful, for it provides a recipe for constructing $U$ in terms of the give items $\left(B_{n}\right)_{n \in N},\left(V_{n}\right)_{n \in N}$ and $H$. In the measure theoretic context [14], this recipe has been used to some advantage for the study of Banach space valued strongly countably additive vector measures; see Note (b) following Theorem 2.3. 
(b) In the context of general linear operators, the formulation of Theorem 2.2 for sets of operators (as opposed to a single one) also will prove particularly useful: in $\S 4$ it will be used to characterize compact sets of compact operators on Banach spaces.

Some special cases of Theorem 2.2 for a single linear operator are specified next:

THEOREM 2.3 [33]. If $X$ is a quasinormable les with property (cnc), and $(Y, \rho)$ is a sequentially complete les with a metrizable lc topology $\rho_{1}$ finer than $\rho$, then every $\rho_{1}$-continuous linear operator from $X$ into $Y$, which transforms bounded sets into precompact (resp. weakly relatively compact) subsets of $(Y, \rho)$, is a precompact (resp. weakly compact) operator from $X$ into $(Y, \rho)$.

In particular, every continuous linear operator from a Schwartz (resp. semi-reflexive) gDF space into a Fréchet space is compact (resp. weakly compact).

Notes and first applications. (a) This special case of Theorem 2.2 contains the above results of Grothendieck, van Dulst and Sentilles. Note that the very last statement of Theorem 2.3 can be viewed as an extension of the (trivial) fact, that every continuous linear operator on a reflexive Banach space is weakly compact, to the case of semireflexive $\mathrm{gDF}$ spaces, with the specified restriction on the range spaces.

(b) The applicability of Theorem 2.3 to the strict topologies mentioned in $\S 1$ has been pointed out already in [33]. A further concrete situation for which Theorems 2.2 and 2.3 provide new tools, is Graves' [13] "linearization of vector measures": For a $\sigma$-algebra $\Sigma$ of subsets of a set $S$ and a Banach space $X$, the space of bounded vector measures from $\Sigma$ into $X$ is in one-to-one correspondence with the continuous linear operators from the space $\mathscr{S}(\Sigma)$ of $\Sigma$-simple functions, endowed with the sup-norm topology, into $X: \mu \mapsto$ integration with respect to $\mu$. W. H. Graves in [13] specified an lc topology $\tau$ on $\mathscr{S}(\Sigma)$, coarser than the sup-norm topology, which singles out the strongly countably additive vector measures as exactly those whose associated operators are $\tau$-continuous. $(\mathscr{S}(\Sigma), \tau)$ is gDF [13, Thm. 2.2, p. 12], and its completion $(\widetilde{\mathscr{S}(\Sigma)}, \widetilde{\tau})$ is semi-reflexive [13, Thm. 10.5, p. 53]. In this way, strongly countably additive vector measures into a Fréchet space $X$ come out to be just continuous linear operators from the semi-reflexive gDF space $(\widetilde{\mathscr{S}(\Sigma)}, \tilde{\tau})$ into $X$. Theorem 2.3 thus reveals that the associated operators not only transform the sup-norm unit ball into a weakly relatively compact 
set (weak relative compactness of ranges of sca vector measures), but also a certain $\tau$-zero neighbourhood. In [14], proposition 3. of Theorem 2.2 is used to specify such a zero neighbourhood in terms of the measure $\mu$ [14, Thms. 10 and 11]. (Further applications of $\mathrm{gDF}$ techniques in the context of vector measures are to be found in $[15]$.)

Proof of Theorem 2.2. The proof consists of two steps: First it is shown:

(1) There exists a zero neighbourhood $U$ in $X$ and a sequence $\left(C_{n}\right)_{n \in N}$ of $\rho$-precompact (rest. $\rho$-weakly relatively compact) disks in $Y$ such that $H(U) \subset \cap\left\{C_{n}+V_{n} \mid n \in N\right\}$, where $\left(V_{n}\right)_{n \in N}$ is a (decreasing) $\rho_{1}$-zero neighbourhood base in $Y$.

Proof.

(i) There exist zero neighbourhoods $U_{n}^{\prime}$ in $X$ such that $H\left(U_{n}^{\prime}\right) \subset$ $V_{n}, n \in N$ ( $\rho_{1}$-equicontinuity of $H$ ).

(ii) There exist zero neighbourhoods $U_{n}$ in $X$ with the property that, for all $\alpha>0$, there exists $B_{\alpha}^{n}$ bounded in $X$ such that $U_{n} \subset$ $\alpha U_{n}^{\prime}+B_{\alpha}^{n}$ (quasinormability of $X$ ).

(iii) $U=\cap\left\{\alpha_{n} U_{n} \mid n \in N\right\}$ is a zero neighbourhood in $X$ for a suitable sequence $\left(\alpha_{n}\right)_{n \in N}$ of positive reals $((\mathrm{chc})$ for $X)$. Thus, for $n \in N: H(U) \subset \alpha_{n} H\left(U_{n}\right) \subset\left(U_{n}^{\prime}\right)+H\left(\alpha_{n} B_{\alpha_{n}^{-1}}^{n}\right) \subset H\left(\alpha_{n} B_{\alpha_{n}^{-1}}^{n}\right)+V_{n}$, which proves (1).

Note. Again, based on the more technical result given in Proposition 1.7 instead of Theorem 1.4 itself, proposition (1) could have been derived by a technique analogous to the one used in the proof of Proposition 2.1. The above independent proof, however, keeps things more transparent.

Assertion (1) completes the proof in the precompact case. The "weak case" is completed by means of the following result:

Lemma 2.4. Let $(Y, \rho)$ be a sequentially complete les, $\rho_{1} a$ metrizable lc topology on $Y$, finer than $\rho$, and $\left(V_{n}\right)_{n \in N} a \rho_{1}$-zero neighbourhood base, all $V_{n}$ disks. If $A$ is a subset of $Y$ with the property that, for every $n \in N$, there exists a $\rho$-weakly relatively compact disk $C_{n}$ in $Y$ such that $A \subset C_{n}+V_{n}$, then $A$ is o-weakly relatively compact.

For $\rho=\rho_{1}=$ Banach space topology, this is to be found in [17, $\mathrm{V}, 4.1$, Lemme on p. 296]. Reasoning as in the proof of this result in [17] the Alaoglu-Bourbaki Theorem reveals that it is enough to show that the weak*-closure of $A$ in the $\rho$-bidual of $Y$ is contained in $Y$, for $A$ is $\rho$-bounded. By assumption, we have (bars denoting 
weak*-closure in the $\rho$-bidual of $\left.Y): \bar{A} \subset \cap \overline{\left\{C_{n}+V_{n}\right.} \mid n \in N\right) \subset \cap$ $\left\{C_{n}+\bar{V}_{n} \mid n \in N\right\}$. Hence, for $z \in \bar{A}$, there exist $b_{n} \in Y$ and $v_{n} \in \bar{V}_{n}, n \in N$, such that $z=b_{n}+v_{n}$ for all $n \in N$. For $V$ a $\rho$-zero neighbourhood, there exists $n_{0} \in N$ such that $V_{n} \subset V$ for all $n>n_{0}$. It follows that $z-b_{n}=v_{n} \in \bar{V}_{n} \subset \bar{V}$ for all $n>n_{0}$. This shows that $\left(b_{n}\right)_{n \in N}$ is a $\rho$ Cauchy sequence in $Y$. $(Y, \rho)$ being sequentially complete, $\left(b_{n}\right)_{n \in N}$ is $\rho$-convergent to an element $y \in Y$. It is now easy to conclude that $z=y \in Y$, which completes the proof.

The proof of Proposition 3 of Theorem 2.2 now follows from two observations:

(i) $U=\cap\left\{n B_{n}+H^{(-1)}\left(V_{n}\right) \mid n \in N\right\}$ is a zero neighbourhood in $X$ ( $X$ is gDF and $H$ is $\rho_{1}$-equicontinuous), and

(ii) $H(U) \subset \cap\left\{H\left(n B_{n}\right)+V_{n} \mid n \in N\right\}$, which is proposition (1) of the proof just given.

A particular application of the above results to general [weakly] compact operators is the following extension of a well known Banach space result:

Proposition 2.5. Let $X$ be a gDF space and $Y$ a Fréchet space. The spaces $K(X, Y)$ and $W(X, Y)$ of compact and of weakly compact linear operators from $X$ into $Y$, respectively, are closed linear subspaces of the space $L_{b}(X, Y)$ of continuous linear operators from $X$ into $Y$, endowed with the topology of uniform convergence on the bounded subsets of $X$. In particular, $K_{b}(X, Y)$ and $W_{b}(X, Y)$ are Fréchet spaces.

Proof. Whenever $u \in L(X, Y)$ is the $b$-limit of a sequence $\left(u_{n}\right)_{n \in N}$ in $L(X, Y)$, then, given a bounded subset $B$ of $X$, for every zero neighbourhood $V$ in $Y$, there exists $n \in N$ such that $u(B) \subset u_{n}(B)+V$. This shows that $u$ transforms bounded sets into [weakly] relatively compact ones, provided that all $u_{n}$ 's are [weakly] compact (for the weak case, again use Lemma 2.4). Theorem 2.3 now yields the desired conclusion.

Before turning to further applications of Theorems 2.2 and 2.3, we conclude this section with a discussion of two more classes of gDF spaces.

Whenever $X$ is an les whose strong dual is Fréchet, then $X_{c}^{\prime \prime}$ (resp. $X_{w c}^{\prime \prime}$ ) is semi-Montel gDF (resp. semi-reflexive gDF). Hence, by Theorem 2.3, every continuous linear operator from $X_{c}^{\prime \prime}$ (resp. $X_{w_{c}}^{\prime \prime}$ ) into a Fréchet space is compact (resp. weakly compact). Exactly the same is true for the particular linear subspace $X_{c}$ (resp. $X_{w c}$ ). But more can be said: $X_{c}$ and $X_{w c}$ are even $\mathrm{gDF}$.

Proposition 2.6. Let $X$ be an les whose strong dual is Fréchet, 
and denote by $X_{c}\left[X_{w c}\right]$ the space $X$, endowed with the topology of uniform convergence on the $\left[\right.$ weakly $\left.=\sigma\left(X^{\prime}, X^{\prime \prime}\right)\right]$ compact disks of $X_{b}^{\prime}$. Then the spaces $X_{c}, X_{w c}$ and $\left(X, \tau\left(X, X^{\prime}\right)\right)$ are $\mathrm{gDF}$, and every continuous linear operator from $X_{c}\left[X_{w c}\right]$ into a Fréchet space is [weakly] compact.

Proof. The gDF property of $X$ with the Mackey topology is immediate from the assumption. For a proof of the gDF property for the other two spaces, it has to be shown that a linear operator from any of them into a Banach space is continuous as soon as its restrictions to the bounded sets are. Let $\left(B_{n}\right)_{n \in N}$ be a fundamental sequence of bounded sets in $(X, \tau)$, all $B_{n}$ disks, $B_{n}+B_{n} \subset B_{n+1}, Y$ a Banach space, and $u$ a linear operator from $X$ into $Y$.

Case " $w c$ ". If the restrictions of $u$ to the $B_{n}$ 's are $w c$-continuous, then $u$ is continuous from $\left(X, \tau\left(X, X^{\prime}\right)\right)$ into $Y$, for $\sigma\left(X, X^{\prime}\right) \subset w c \subset$ $\tau\left(X, X^{\prime}\right)$, and the latter topology is gDF. Plain duality implies that $u^{\prime \prime}$ is continuous from $\left(X^{\prime \prime}, \tau\left(X^{\prime \prime}, X^{\prime}\right)\right)$ into $\left(Y^{\prime \prime}, \tau\left(Y^{\prime \prime}, Y^{\prime}\right)\right)$. But the range of $u^{\prime \prime}$ is contained in $Y$ : for $x^{\prime \prime} \in X^{\prime \prime}$, there exists $n \in N$ and a net $\left(x_{\lambda}\right)_{\lambda \in A} \subset B_{n}$ which is $\tau\left(X^{\prime \prime}, X^{\prime}\right)$-convergent to $x^{\prime \prime}$. By assumption on $u$, the net $\left(u x_{\lambda}\right)_{\lambda \in A}$ is norm convergent to some $y \in Y$. Clearly, $u^{\prime \prime} x^{\prime \prime}=y \in Y$.

In this way, $u^{\prime \prime}$ comes out to be a closed graph linear map from the gDF space $\left(X^{\prime \prime}, \tau\left(X^{\prime \prime}, X^{\prime}\right)\right)$ into $Y$, which transforms bounded sets $\left(\sigma\left(X^{\prime \prime}, X^{\prime}\right)\right.$-closures of the $B_{n}$ 's in $\left.X^{\prime \prime}\right)$ into weakly relatively compact sets. Proposition 3.4 of [31] implies that $u^{\prime \prime}$ is weakly continuous, and hence continuous, from $\left(X^{\prime \prime}, \tau\left(X^{\prime \prime}, X^{\prime}\right)\right)$ into $Y$.

Case "c". If the restrictions of $u$ to the bounded sets are $c$ continuous, then they are $w c$-continuous as well, and thus the range of $u^{\prime \prime}$ is contained in $Y$. Again, plain duality implies that $u^{\prime \prime}$ is continuous from $X_{c}^{\prime \prime}$ into $Y_{c}$, and hence closed graph from the gDF space $X_{c}^{\prime \prime}$ into $Y$. Moreover, $u^{\prime \prime}$ transforms bounded sets (c-closures of the $B_{n}$ 's in $X^{\prime \prime}$ ) into relatively compact subsets of $Y$ : the restrictions of $u$ to the $B_{n}$ 's are even $c$-uniformly continuous into $Y$, and the $B_{n}$ 's are $c$-precompact. This time, Proposition 3.4 of [31] directly reveals that $u^{\prime \prime}$ is continuous from $X_{c}^{\prime \prime}$ into $Y$.

[Weak] Compactness of continuous linear operators on $X_{c}\left[X_{w c}\right]$ : Whenever $u$ is a continuous linear operator from $X_{c}\left[X_{w c}\right]$ into a Fréchet space $Y$, then it has a unique continuous linear extension $u$ to the completion $X_{c}^{\prime \prime}\left[X_{w c}^{\prime \prime}\right]$. But $X_{c}^{\prime \prime}$ is semi-Montel $\mathrm{gDF}$, and $X_{w c}^{\prime \prime}$ is semi-reflexive gDF. The desired conclusions follow from Theorem 2.3.

3. Extensions of Schauder's and Gantmaher's theorems, and 
tensor product representations of $K(X, Y)$ and its dual. The basic idea of Theorem 2.3 of the foregoing section is to conclude [weak] compactness of a linear operator $u$ from the (formally) weaker assumption of $u$ being continuous and transforming bounded sets into [weakly] relatively compact sets. In this section, a different direction of thought will be pursued: roughly, it will be shown that such operators are [weakly] compact not only for the original topology of the domain space but also for an even coarser lc topology (Thm. 3.1 below). A particular consequence will be a new representation for the space $K(X, Y)$ of compact linear operators and of its strong dual $K^{\prime}(X, Y)$, for $X$ and $Y$ Banach, or, more generally, for $X \mathrm{gDF}$ and $Y$ Fréchet (Thms. 3.3 and 3.4 below).

Starting point is the following extension to the gDF- $F$-situation, together with a refinement to coarser lc topologies, of Schauder's and Gantmaher's Theorems:

THEOREM 3.1. Let $(X, \tau)$ and $Y$ be les such that $Y$ and the strong dual $X_{b}^{\prime}$ of $(X, \tau)$ are Fréchet, and let $u \in L((X, \tau), Y)$.

(a) The following are equivalent:

(1) $u$ transforms bounded sets into relatively compact sets.

(2) $u^{\prime}$ is compact from $Y_{c}^{\prime}$ into $X_{b}^{\prime}$.

(3) $u^{\prime}$ is compact from $Y_{b}^{\prime}$ into $X_{b}^{\prime}$.

(4) $u$ is compact from $X_{c}$ into $Y$.

In particular, if, in addition, $(X, \tau)$ is sequentially evaluable, then all four propositions are equivalent to

(5) $u$ is compact from $(X, \tau)$ into $Y$.

(b) The following are equivalent:

(1) $u$ transforms bounded sets into weakly relatively compact sets.

(2) $u^{\prime}$ is weakly compact from $Y_{w_{c}}^{\prime}$ into $X_{b}^{\prime}$.

(3) $u^{\prime}$ is weakly compact from $Y_{b}^{\prime}$ into $X_{b}^{\prime}$.

(4) $u$ is weakly compact from $X_{w c}$ into $Y$.

In particular, if, in addition, $(X, \tau)$ is $\mathrm{gDF}$, then all four propositions are equivalent to

(5) $u$ is weakly compact from $(X, \tau)$ into $Y$.

Of particular interest is the special case where $X$ and $Y$ are Banach spaces (in accordance with the usual Banach space notation, the topological dual of a normed space $Z$ will be denoted by $Z^{*}$ ):

TheOREm 3.2. Let $X$ be a normed space, $Y$ a Banach space, and let $u \in L(X, Y)$.

(a) The following are equivalent:

(1) $u$ is compact. 
(2) $u^{*}$ is compact.

(3) $u^{*}$ is compact weak*-weakly $\left(\sigma\left(Y^{*}, Y\right)-\sigma\left(X^{*}, X^{* *}\right)\right)$ continuous.

( $\left.3^{\prime}\right) \quad u^{*}$ is compact from $Y_{c}^{*}$ into $X^{*}$, i.e., there exists $K$ compact in $Y$ such that $u^{*}\left(K^{0}\right)$ is relatively compact in $X^{*}$.

(4) $u$ is compact from $X_{c}$ into $Y$, i.e., there exists $K$ compact in $X^{*}$ such that $u\left(K^{0}\right)$ is relatively compact in $Y$.

(b) The following are equivalent:

(1) $u$ is weakly compact.

(2) $u^{*}$ is weakly compact.

(3) $u^{*}$ is weakly compact and weak*-weakly $\left(\sigma\left(Y^{*}, Y\right)-\sigma\left(X^{*}\right.\right.$, $\left.X^{* *}\right)$ ) continuous.

( $\left.3^{\prime}\right) u^{*}$ is weakly compact from $Y_{w c}^{*}$ into $X^{*}$, i.e., there exists $C$ weakly compact in $Y$ such that $u^{*}\left(C^{0}\right)$ is weakly $\left(\sigma\left(X^{*}, X^{* *}\right)\right)$ relatively compact in $X^{*}$.

(4) $u$ is weakly compact from $X_{w c}$ into $Y$, i.e. there exists $C$ weakly $\left(\sigma\left(X^{*}, X^{* *}\right)\right)$ compact in $X^{*}$ such that $u\left(C^{0}\right)$ is weakly relatively compact in $Y$.

Notes. (a) The following particular result is included in proposition (a) of Theorem 3.1:

Every continuous linear operator from a sequentially evaluable les with a fundamental sequence of bounded sets into a Fréchet space, which tranforms bounded sets into relatively compact sets, is compact.

It is not clear whether this variant of the theme of $\S 2$ is covered by Theorem 2.3, for it is not known whether the (cnc) property and quasinormability hold for the spaces just specified. An example of such a space which is not $\mathrm{gDF}$ has been exhibited by M. Valdivia (oral communication by $H$. Jarchow). Note that, for a gDF space $(X, \tau)$, all spaces $(X, \rho)$, with $\rho$ an lc topology between the $c$-topology and the Mackey topology $\tau\left(X, X^{\prime}\right)$, are sequentially evaluable. Also note at this point that, for an lcs with a fundamental sequence of precompact sets, the properties of being sequentially evaluable and of being $\mathrm{gDF}$ are equivalent.

(b) Proposition (a) of Theorem 3.2 is implicit in Grothendieck's early work in functional analysis: compactness of $u$ translates by polarity into continuity of $u^{*}$ from $Y_{c}^{*}$ into $X^{*}$. Compactness of $u^{*}$ for these topologies then follows from Grothendieck's result [16, Cor. 1 of Thm. 11, p. 114] (see the beginning of section 2 above) and the fact that $Y_{0}^{*}$ is a Schwartz space, the latter being a consequence of the Banach-Dieudonné Theorem. Equivalent formulations of prop- 
osition (a), in particular, the coincidence of compact operators with the quasi- $\infty$-nuclear operators of Persson/Pietsch [28, p. 56], have been given in [43, Thm. 1] and in [29].

Proof of Theorem 3.1. Case (a): The assumption of (1) (resp. of (3)) translates by plain duality techniques into $u^{\prime}$ (resp. $u^{\prime \prime}$ ) being continuous from $Y_{c}^{\prime}$ into $X_{b}^{\prime}$ (resp. from $X_{c}^{\prime \prime}$ into $Y_{b}^{\prime \prime}$ ). In both cases, Theorem 2.3 reveals the compactness of the respective mappings for these topologies. This, in turn, implies (2) (resp. (4)). Finally, whenever $(X, \tau)$ is sequentially evaluable, then $\tau$ is finer than the $c$-topology, and (5) is implied by (4). Case (b): Proceeding as in the proof of (a), the assumption of (1) (resp. (3)) translates into $u^{\prime}$ (resp. $u^{\prime \prime}$ ) being continuous from $Y_{w c}^{\prime}$ into $X_{b}^{\prime}$ (resp. from $X_{w c}^{\prime \prime}$ into $\left.Y_{b}^{\prime \prime}\right)$. Again, the weak compactness of the respective mappings for these topologies, and thus (2) (resp. (4)), is a consequence of Theorem 2.3. Finally, whenever $(X, \tau)$ is $\mathrm{gDF}$, then, according to just this theorem, (1) and (5) are equivalent.

Theorem 3.1 is a useful tool for the investigation both of individual [weakly] compact operators (factorization, representation) and of the whole space of [weakly] compact operators, see [35] for a survey. Confining ourselves here just to the space $K(X, Y)$ of compact operators, one of the most fruitful consequences of Theorem 3.1 are the following new tensor product representations of $K(X, Y)$ and of its dual:

THEOREM 3.3 Let $X$ be a gDF space and $Y$ a Fréchet space.

(a) The Fréchet space $K_{b}(X, Y)$ of compact linear operators from $X$ into $Y$ with the topology of uniform convergence on the bounded subsets of $X$ is topologically isomorphic to the strong dual of the projective tensor product space $X_{c}^{\prime \prime} \widetilde{\otimes}_{\pi} Y_{c}^{\prime}$.

(b) The dual space $K_{b}^{\prime}(X, Y)$ of the space $K_{b}(X, Y)$ is algebraically isomorphic to the projective tensor product space $X_{c}^{\prime \prime} \widetilde{\otimes}_{\pi} Y_{c}^{\prime}$. Topologically, this latter space is exactly the c-dual of $K_{b}(X, Y)$.

Of particular interest is the special case of Banach spaces:

THeOREM 3.4. Let $X$ and $Y$ be Banach spaces.

(a) $K(X, Y)$ with the operator norm is isometrically isomorphic to the dual space of the (locally convex) projective tensor product space $X_{c}^{* *} \widetilde{\otimes}_{\pi} Y_{c}^{*}$, endowed with the (Banach space) topology of uniform

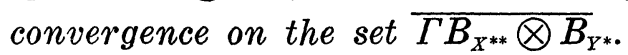

(b) $K^{*}(X, Y)$ is isometrically isomorphic to the space $X_{c}^{* *} \widetilde{\otimes}_{\pi} Y_{c}^{*}$ 
with norm unit ball $\overline{\Gamma B_{X^{* *}} \otimes B_{Y^{*}}}$.

Proof of Theorem 3.3. (a): For a given $u \in K(X, Y)$, Theorem 3.1 assures the existence of $K_{1}$ compact in $X_{b}^{\prime}$ and $K_{2}$ compact in $Y$ such that $u^{\prime \prime}\left(K_{1}^{0}\right) \subset K_{2}$. In this way, the associated bilinear form $B_{u}: X^{\prime \prime} \times Y^{\prime} \rightarrow K$, defined by $B_{u}\left(x^{\prime \prime}, y^{\prime}\right)=\left\langle u^{\prime \prime} x^{\prime \prime}, y^{\prime}\right\rangle$, turns out to be continuous from $X_{c}^{\prime \prime} \times Y_{c}^{\prime}$ into $K$. This shows that the correspondence $u \mapsto B_{u}$ is a topological isomorphism from $K_{b}(X, Y)$ onto $B_{b b}\left(X_{c}^{\prime \prime}, Y_{c}^{\prime}\right)$. But Theorem 1.9 reveals that the latter space is just the strong dual of $X_{c}^{\prime \prime} \otimes_{\pi} Y_{c}^{\prime}$.

(b): According to Corollary 1.12 , the space $X_{c}^{\prime \prime} \tilde{\bigotimes}_{\pi} Y_{c}^{\prime}$ is semiMontel, and thus also semi-reflexive. Together with Proposition (a), this proves the first assertion of (b). The second one follows again from Corollary 1.12, and the fact [31, Thm. 3.8] that any two semiMontel gDF topologies which are comparable already must be identical.

Finally, the additional information on the isometries in Theorem 3.4 is provided by Theorem 1.9 .

Notes. (a) Theorem 3.4 is at the base of a unified approach to a broad variety of structural properties of the space $K(X, Y)$ and its dual: compactness, weak compactness and weak convergence in $K(X, Y)$, reflexivity of $K(X, Y)$, geometric properties of $K^{*}(X, Y)$ etc. In joint papers with H. S. Collins $[6,7]$, this program is carried out in detail. For a survey of the results, consult [8]. A characterization of compactness in $K(X, Y)$ is the object of $\S 4$.

(b) The proof of part (a) of Theorem 3.3 above reveals that for Banach spaces $X$ and $Y$, and an operator $u \in K(X, Y)$, the associated bilinear form $B_{u}$ on $X^{\prime \prime} \times Y^{\prime}$, defined by $B_{u}\left(x^{\prime \prime}, y^{\prime}\right)=\left\langle u^{\prime \prime} x^{\prime \prime}, y^{\prime}\right\rangle$, is continuous from $X_{c}^{\prime \prime} \times Y_{c}^{\prime}$ into $K$. Thus, according to the compactness of $B_{X^{\prime \prime}} \times B_{Y^{\prime}}$ in $X_{c}^{\prime \prime} \times Y_{c}^{\prime}$, there exist $x^{\prime \prime} \in B_{X^{\prime \prime}}$ and $y^{\prime} \in B_{Y^{\prime}}$ such that $\|u\|=\left\langle u^{\prime \prime} x^{\prime \prime}, y^{\prime}\right\rangle$. We conclude that every compact linear operator from a Banach space $X$ into a Banach space $Y$ attains its norm on $B_{X^{\prime \prime}}$. This shows that in the corresponding result of Baker [1, Thm. $1(\mathrm{i})]$, the assumption of $B_{X^{\prime \prime}}$ being weak* $=\sigma\left(X^{\prime \prime}, X^{\prime}\right)$-sequentially compact is superfluous.

4. Compactness in $K(X, Y)$. A particular example of the range of applicability of the techniques developed so far is the following characterization of (operator norm) compact sets of compact operators:

THEOREM. Let $X$ be a normed space and $Y$ a Banach space. Then, for a subset $H$ of $K(X, Y)$, the following are equivalent:

(1) $H$ is relatively compact (in the operator norm). 
(2) $H B_{X}$ and $H^{*} B_{Y^{*}}$ are relatively compact in $Y$ and $X^{*}$, respectively.

(3) $H B_{X}$ is relatively compact in $Y$, and $H^{*}\left(y^{*}\right)$ is relatively compact in $X^{*}$ for all $y^{*} \in Y^{*}$.

(4) $H^{*} B_{Y^{*}}$ is relatively compact in $X^{*}$, and $H(x)$ is relatively compact in $Y$ for all $x \in X$.

(5) There exists $K$ compact in $X^{*}$ such that $H\left(K^{0}\right)$ is relatively compact in $Y$.

Notes. (a) The equivalence of (1) and (3) and (4) is a result of Palmer's [27, Thms. 2.1 and 2.2]. In the presence of the approximation property for either $X^{*}$ or $Y$, the equivalence of (1) and (2) has been proved by Holub [22, Cor. to Thm. 1].

Finally, the equivalence of (1), (2) and (5) can also be deduced from L. Schwartz' $\varepsilon$-product techniques [38; especially I. 1, Prop. 2, p. 22, and Prop. 10, p. 45], together with Theorem 1.4; see [35] for details.

(b) Together with the Davis/Figiel/Johnson/Pelczynski factorization theorem for weakly compact operators [9], the equivalence of (1) and (5) can be used to factor an operator norm convergent sequence of compact operators through one and the same reflexive Banach space in such a way, that the convergence of the sequence even takes place for the respective new (stronger) operator norm. Problems of this kind are being discussed in [36, 37].

Proof of the Theorem. First, recall the following isometric isomorphisms (Theorem 3.4):

$$
K(X, Y)=B\left(X_{c}^{* *}, Y_{c}^{*}\right)=\left(X_{c}^{* *} \bigotimes_{\pi} Y_{c}^{*}\right)_{b}^{\prime} .
$$

Since $X_{c}^{* *} \tilde{\bigotimes}_{\pi} Y_{c}^{*}$ is semi-Montel gDF, its $c$-topology coincides with its original topology, i.e., the equicontinuous and the strongly relatively compact subsets of its dual coincide. Hence, the equicontinuous and the relatively compact subsets of $B_{b b}\left(X_{c}^{* *}, Y_{c}^{*}\right)$ coincide. Furthermore, according to Theorem 1.4, they are the same as the equihypocontinuous subsets. Together with (*), this establishes the equivalence of (1), (2) and (5).

The first condition of proposition (3) (resp. of (4)) means that $H^{*}$ (resp. $H$ ) is equicontinuous from $Y_{c}^{*}$ into $X^{*}$ (resp. from $X_{c}$ into $Y$ ). According to a consequence of the Arzela-Ascoli Theorem (c.f. [17, 0.7, Cor. 2 of Thm. 6, p. 17]), $H^{*} \subset L\left(Y_{c}^{*}, X^{*}\right)$ (resp. $H \subset L\left(X_{c}, Y\right)$ ) is precompact with respect to the topology of uniform convergence on the precompact subsets of $Y_{c}^{*}$ (resp. of $X_{c}$ ), if and only if $H^{*} \mid P$ (resp. $H \mid P$ ) is equicontinuous for all $P$ precompact in $Y_{c}^{*}$ (resp. in 
$X_{c}$ ), and $H^{*}\left(y^{*}\right)$ (resp. $H(x)$ ) is precompact in $X^{*}$ (resp. in $Y$ ) for all $y^{*} \in Y^{*}$ (resp. for all $x \in X$ ). Since $Y_{c}^{*}$ and $X_{c}$ are gDF spaces whose bounded sets are precompact (Examples 2 in $\S 1$, and Proposition 2.6), the equivalence of propositions (1), (3) and (4) is now apparent. This completes the proof.

\section{REFERENCES}

1. J. M. Baker, $A$ note on compact operators which attain their norm, Pacific J. Math., 82 (1979), 319-321.

2. K.-D. Bierstedt and R. Meise, Induktive Limites gewichteter Räume stetiger und holomorpher Funktionen, J. Reine Angew. Math., 282 (1976), 186-220.

3. K. Brauner, Duals of Fréchet spaces and a generalization of the Banach-Dieudonné Theorem, Duke Math. J., 40 (1974), 845-855.

4. R. C. Buck, Bounded continuous functions on a locally compact space, Mich. Math. J., 5 (1958), 95-104.

5. R. C. Busby, Double centralizers and extensions of $C^{*}$-algebras, Trans. Amer. Math. Soc., 132 (1968), 79-88.

6. H. S. Collins and W. Ruess, Weak compactness in spaces of compact operators, preprint 1980.

7. - Duals of spaces of compact operators, to appear in Studia Math.

8. - Duality for spaces of compact operators, preprint 1980.

9. W. J. Davis, T. Figiel, W. B. Johnson and A. Pelczynski, Factoring weakly compact operators, J. Functional Analysis, 17 (1974), 311-327.

10. D. van Dulst, (Weakly) Compact mappings into (F) spaces, Math. Ann., 224 (1976), 111-115.

11. D. H. Fremlin, D. J. H. Garling and R. Haydon, Bounded measures on topological spaces, Proc. London Math. Soc., (1972), 115-136.

12. D. J. H. Garling, A generalized form of inductive-limit topology for vector spaces, Proc. London Math. Soc., 14, (1964) 1-28.

13. W. H. Graves, On the theory of vector measures, Memoirs Amer. Math. Soc., 195 (1977).

14. W. H. Graves and W. Ruess, Compactness in spaces of vector-valued measures, and a natural Mackey topology for spaces of bounded measurable functions, Amer. Math. Soc. Contemporary Math. 2, Amer. Math. Soc., Providence, RI (1980).

15. - Compactness and weak compactness in spaces of compact range vector measures, preprint 1980.

16. A. Grothendieck, Sur les espaces $(F)$ et $(D F)$, Summa Brasil. Math., 3 (1954), 57-122.

17. _ Espaces vectoriels topologiques, Sao Paulo 1954.

18. - Produits tensoriels topologiques et espaces nucléaires, Memoirs Amer. Math. Soc., 16 (1955).

19. S. Heinrich, Approximation properties in tensor products, Math. Notes 17 (1975), 269-272 (1975), (translated from Mat. Zametki 17 (1975), 459-466).

20. R. Hollstein, $\sigma$-lokaltopologische Räume und projektive Tensorprodukte, collectanea Math., 26 (1975), 3-16.

21. — $(D C F)-R \ddot{a} u m e$ und lokalkonvexe Tensorprodukte, Arch. Math. 29 (1977), 524-531.

22. J. R. Holub, Compactness in topological tensor products and operator spaces, Proc. Amer. Math. Soc., 36 (1972), 398-406.

23. J. Horváth, Topological Vector Spaces and Distributions I, Reading, Massachusetts; Addison-Wesley 1966. 
24. K. Noureddine, Espaces $d u$ type $D_{b}$, C. R. Acad. Sc. Paris, 276 (1973), A1301A1303.

25. - Nouvelles classes d'espaces localement convexes, Publ. Dépt. Math. Lyon, 10, (1973), 105-123.

26. ㄴ. Note sur les espaces $D_{b}$, Math. Ann. 219 (1976), 97-103.

27. T. W. Palmer, Totally bounded sets of precompact linear operators. Proc. Amer. Math. Soc., 20 (1969), 101-106.

28. A. Persson, A. Pietsch, p-nukleare und p-integrale Abbildungen in Banach-Räumen, Studia Math., 33 (1969), 19-61.

29. D. J. Randtke, $A$ factorization theorem for compact operators, Proc. Amer. Math. Soc., 34 (1972), 201-202.

30. W. Roelcke, On the finest locally convex topology agreeing with a given topology on a sequence of absolutely convex sets. Math. Ann. 198 (1972), 57-80.

31. W. Ruess, Closed graph theorems for generalized inductive limit topologies, Math. Proc. Cambridge Phil. Soc., 82 (1977), 67-83.

32. - Halbnorm-Dualität und induktive Limestopologien in der Theorie lokalkonvexer Räume, Habilitationsschrift, Bonn 1976.

33. - On the locally convex structure of strict topologies, Math. Z., 153 (1977), 179-192.

34. - The strict topology and DF spaces, Proc. Conf. Fctal. Analysis, Paderborn 1976. North-Holland Math. Studies 27 (1977), 105-118.

35. - Spaces of compact operators on Banach spaces, Special topics of applied mathematics. Frehse/Pallaschke/Trottenberg (eds.). North-Holland Publ. Comp. (1980). 36. Compactness and collective compactness in spaces of compact operators, to appear in F. Math. Anal. Appl.

37. W. Ruess and F. D. Sentilles. Notes on factoring [weakly] compact operators, unpublished manuscript.

38. L. Schwartz, Théorie des distributions à valeurs vectorielles I, Ann. Inst. Fourier, 7 (1957), 1-139.

39. F. D. Sentilles, Compact and weakly compact operators on $C(S)_{\beta}$, Illinois J. Math., 13 (1969), 769-776.

40. - Bounded continuous functions on a completely regular space, Trans. Amer. Math. Soc., 168 (1972), 311-336.

41. - An $L^{1}$-space for Boolean algebras and semi-reflexivity of spaces $L^{\infty}(X, \Sigma, \mu)$, Trans. Amer. Math. Soc., 226 (1977) 1-37.

42. F. D, Sentilles and D. C. Taylor, Factorization in Banach algebras and the general strict topology, Trans. Amer. Math. Soc., 142 (1969), 141-152.

43. T. Terzioglu, A characterization of compact linear mappings, Arch. Math., 22 (1971) 76-78.

44. J. H. Webb, Sequential convergence in locally convex spaces, Proc. Cambridge Phil. Soc., 64 (1968), 341-364.

Received March 14, 1980 and in revised form November 11, 1980. This paper is based on Chapters II and IV of the author's Habilitationsschrift [32].

UNIVERSITAT ESSEN

UNIVERSITATSSTR, 3

43 Essen 1, Federal Republic of Germany 



\title{
PACIFIC JOURNAL OF MATHEMATICS
}

\section{EDITORS}

\author{
DONALD BABBITT (Managing Editor) \\ University of California \\ Los Angeles, CA 90024 \\ Hugo RossI \\ University of Utah \\ Salt Lake City, UT 84112 \\ C. C. MOORE and ANDREW OGG \\ University of California \\ Berkeley, CA 94720
}

J. DugundjI

Department of Mathematics

University of Southern California

Los Angeles, CA 90007

R. FinN and J. Milgram

Stanford University

Stanford, CA 94305

\section{ASSOCIATE EDITORS}
R. ARENS
E. F. Beckenbach
B. H. NeumanN
F. WOLF
K. YoSHIDA

\section{SUPPORTING INSTITUTIONS}

\author{
UNIVERSITY OF ARIZONA \\ UNIVERSITY OF BRITISH COLUMBIA \\ CALIFORNIA INSTITUTE OF TECHNOLOGY \\ UNIVERSITY OF CALIFORNIA \\ MONTANA STATE UNIVERSITY \\ UNIVERSITY OF NEVADA, RENO \\ NEW MEXICO STATE UNIVERSITY \\ OREGON STATE UNIVERSITY
}

\author{
UNIVERSITY OF OREGON \\ UNIVERSITY OF SOUTHERN CALIFORNIA \\ STANFORD UNIVERSITY \\ UNIVERSITY OF HAWAII \\ UNIVERSITY OF TOKYO \\ UNIVERSITY OF UTAH \\ WASHINGTON STATE UNIVERSITY \\ UNIVERSITY OF WASHINGTON
}

The Supporting Institutions listed above contribute to the cost of publication of this Journal, but they are not owners or publishers and have no responsibility for its content or policies.

Mathematical papers intended for publication in the Pacific Journal of Mathematics should be in typed form or offset-reproduced, (not dittoed), double spaced with large margins. Please do not use built up fractions in the text of the manuscript. However, you may use them in the displayed equations. Underline Greek letters in red, German in green, and script in blue. The first paragraph or two must be capable of being used separately as a synopsis of the entire paper. Please propose a heading for the odd numbered pages of less than 35 characters. Manuscripts, in triplicate, may be sent to any one of the editors. Please classify according to the scheme of Math. Reviews, Index to Vol. 39. Supply name and address of author to whom proofs should be sent. All other communications should be addressed to the managing editor, or Elaine Barth, University of California, Los Angeles, California, 90024.

50 reprints to each author are provided free for each article, only if page charges have been substantially paid. Additional copies may be obtained at cost in multiples of 50 .

The Pacific Journal of Mathematics is issued monthly as of January 1966. Regular subscription rate: $\$ 102.00$ a year (6 Vols., 12 issues). Special rate: $\$ 51.00$ a year to individual members of supporting institutions.

Subscriptions, orders for numbers issued in the last three calendar years, and changes of address shoud be sent to Pacific Journal of Mathematics, P.O. Box 969, Carmel Valley, CA 93924, U.S.A. Old back numbers obtainable from Kraus Periodicals Co., Route 100, Millwood, NY 10546.

PUBLISHED BY PACIFIC JOURNAL OF MATHEMATICS, A NON-PROFIT CORPORATION

Printed at Kokusai Bunken Insatsusha (International Academic Printing Co., Ltd.). 8-8, 3-chome, Takadanobaba, Shinjuku-ku, Tokyo 160, Japan. 


\section{Pacific Journal of Mathematics}

\section{Vol. 98, No. 2 \\ April, 1982}

Thomas E. Armstrong, Barycentric simplicial subdivision of infinite-dimensional simplexes and octahedra

Hom Nath Bhattarai and James William Fernandez, Joins of double coset

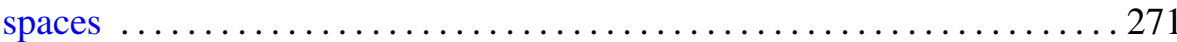

Alexandru Buium, Ritt schemes and torsion theory $\ldots \ldots \ldots \ldots \ldots \ldots \ldots 281$

Jacob Burbea, Operator-valued Pick's conditions and holomorphicity ..... 295

Su-Shing Chen, Duality condition and property $(\mathrm{S}) \ldots \ldots \ldots \ldots \ldots \ldots . \ldots . \ldots 313$

Ky Fan, Evenly distributed subsets of $S^{n}$ and a combinatorial application . . 323

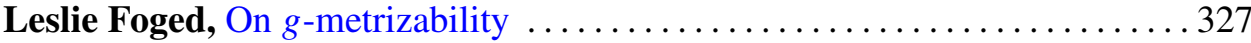

John Groves Heywood, An error estimate uniform in time for spectral

Galerkin approximations of the Navier-Stokes problem ............ 333

Aggie Ho, The Krê̆ n-Milman property and complemented bushes in

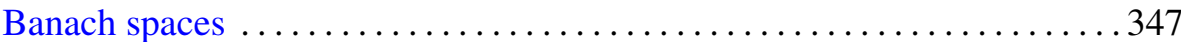

David R. Jackett, Rings on certain mixed abelian groups ............. 365

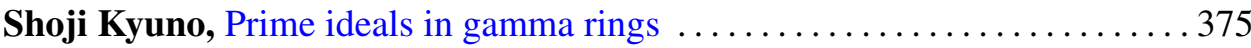

George Lucius O'Brien, Zero-inducing functions on finite abelian

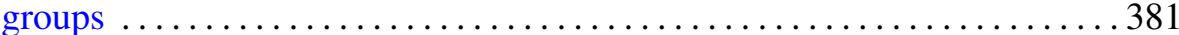

P. Robba, Sur les équations différentielles linéaires $p$-adiques. II ......... 393

Wolfgang Ruess, [Weakly] compact operators and DF spaces ...........419

Claude Schochet, Topological methods for $C^{*}$-algebras. II. Geometry resolutions and the Künneth formula

Harry F. Smith, Jr., Equivalent nilpotencies in certain generalized right

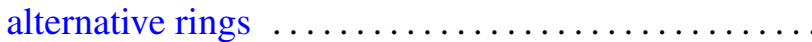

Max Warshauer, Diagonalization up to Witt 469

Hugh C. Williams, A class of primality tests for trinomials which includes the Lucas-Lehmer test 